\title{
THREE-DIMENSIONAL PHASE FIELD MODEL FOR ACTIN-BASED CELL MEMBRANE DYNAMICS
}

\author{
Mohammad Abu Hamed ${ }^{1,2,3, *}$ And Alexander A. Nepomnyashchy ${ }^{1}$
}

\begin{abstract}
The interface dynamics of a 3D cell immersed in a 3D extracellular matrix is investigated. We suggest a 3D generalization of a known 2D minimal phase field model suggested in Ziebert et al. [J. R. Soc. Interface 9 (2012) 1084-1092] for the description of keratocyte motility. Our model consists of two coupled evolution equations for the order parameter and a three-dimensional vector field describing the actin network polarization (orientation). We derive a closed evolutionary integrodifferential equation governing the interface dynamics of a 3D cell. The equation includes the normal velocity of the membrane, its curvature, cell volume relaxation, and a parameter that is determined by the non-equilibrium effects in the cytoskeleton. This equation can be considered as a 3D generalization of the 2D case that was studied in Abu Hamed and Nepomnyashchy [Physica D 408 (2020)].
\end{abstract}

Mathematics Subject Classification. 92C17.

Received August 8, 2021. Accepted September 27, 2021.

\section{INTRODUCTION}

Over the past decade, increasing attention has been paid to the formulation of computational models that describe the motility of $3 \mathrm{D}$ cells that crawl on flat substrate $[7,12]$ or invade $3 \mathrm{D}$ extracellular matrices (ECM) $[11,14,15]$, which is the subject of this paper. This challenge has been mentioned about a decade ago in the review paper [9] as barely started field. Since that time, the keratocyte motility, including the lamellipodium waves dynamics [10], has been rather well explored using experimental and theoretical approaches.

Actin polymerization is a basic mechanism in $2 \mathrm{D}$ cell motility $[6,10]$. When investigating the dynamics of $3 \mathrm{D}$ cell invasion in 3D ECM, one finds that there are several mechanisms or modes of migration that control 3D cell motility, and a cell may switch between them depending on cell intrinsic and extrinsic factors [1]. Among these mechanisms is the generation of protrusive force through hydrostatic pressure. This mechanism is called amoeboid and it does not need actin polymerization at the leading edge to generate protrusions. Another mechanism, which is the subject of this paper, is the lamellipodium-based protrusion in 3D-ECMs which is called mesenchymal. This mechanism is based on actin polarization to form protrusion that enables cell migration in 3D ECMs, and it is analogous to the 2D actin-based cell motility. This migratory mode has been observed in some metastatic cancer cells while moving in complex 3D environments, see [1] and references therein.

Keywords and phrases: Cell motility, Actin self-assembly.

${ }^{1}$ Department of Mathematics, Technion - Israel Institute of Technology, Haifa 32000, Israel.

2 Department of Mathematics, The College of Sakhnin - Academic College for Teacher Education, Sakhnin 30810 , Israel.

3 Department of Mathematics, Gordon College of Education, Haifa 3570503, Israel.

* Corresponding author: mohammad.jaber.a.h@gmail.com

(C) The authors. Published by EDP Sciences, 2021 
Recently a minimal computational phase field model of 3D cell crawling on general substrate topography, not only on flat surface, has been formulated [13]. In their model, the authors assume that the actin exists only near the substrate surface and vanishes far away. Therefore this model is a nontrivial generalization of their $2 \mathrm{D}$ model [18].

In the present paper we suggest another 3D generalization of the original 2D model developed by Aranson and co-workers $[16,18]$ (see also [4]). We consider a 3D cell surrounded by 3D ECM rather than attached to a surface. It is assumed that the actin exists over all the interface of the 3D cell, hence lamellipodia can be created in any directions.

In the framework of our model, the cell geometry is described by a phase field coupled with a three-dimensional vector field of the actin network polarization. Thus, we suggest a model of motility of actin-based 3D cell surrounded by 3D ECM. Relative to other phase field approaches, this model can be considered as a simple minimal model describing the 3D cell motility $[13,17]$.

In the present paper, we consider the case where the cell does not move as a whole but can change its shape. The structure of the paper is as follows: In Section 2 we present the minimal 3D phase field model. In Section 3 we investigate the dynamics of the spherical shape interface. In Section 4 we consider the general shape interface. We derive a closed evolutionary nonlocal equation that describes the interface dynamics at the onset of cell motion when the cell does not moves as a whole. Also, we investigate the stability of the spherical shape membrane. Finally, in Section 5 we present the conclusions.

\section{Formulation of the MATHEMATiCAL MODEL}

Following [18], we adopt a diffuse-interface description of the cell membrane by means of the phase-field variable $u$. Far from the membrane, that variable tends to 1 inside the cell and to 0 outside the cell; across the membrane, it changes smoothly. The position of the membrane is determined by the relation $u=1 / 2$, see Figure 1.

Because the mesenchymal mechanism of cell protrusion is related to the actin polymerization and orientation of filaments, we expect that the description of that process by a vector field $\mathbf{P}$ of polarization can be an essential element of the model. According to experimental observation, actin polymerization takes place near the membrane where the protrusion is developed [8] . In the framework of the diffuse-interface description, $\nabla u$ acts as a source generating the polarization vector. The actin polymerized near the membrane diffuses into the bulk of the cell where it is destroyed. The processes described above can be modelled by the evolution equation,

$$
\mathbf{P}_{t}=D_{p} \nabla^{2} \mathbf{P}-\tau^{-1} \mathbf{P}-\beta \nabla u
$$

where $D_{p}$ is the diffusion coefficient for $\mathbf{P}$ and $\tau^{-1}$ is the inverse time of the degradation of $\mathbf{P}$ inside the cell. It is assumed that $\mathbf{P}$ is concentrated near the cell membrane, therefore

$$
\mathbf{P}(r \rightarrow \infty)=0
$$

where $r$ is the distance from the origin, and it is also negligible inside the cell far from the membrane, see Figure 1. Equation (2.1) is supplemented by some initial distribution of $\mathbf{P}$ with the properties described above.

Let us discuss now the governing equation for $u$. First of all, it has to be compatible with the basic assumptions concerning the field of the variable $u$, which is changed between 0 (on the infinity and far outside the cell) and 1 (far inside the cell). Also, it has to include a number of biological effects: (i) the stiffness of the membrane; (ii) the influence of polarization "pushing" the membrane; (iii) the volume of the cell does not change significantly. All these conditions are satisfied for the equation

$$
u_{t}=D_{u} \nabla^{2} u-(1-u)(\delta-u) u-\alpha \nabla u \cdot \mathbf{P},
$$



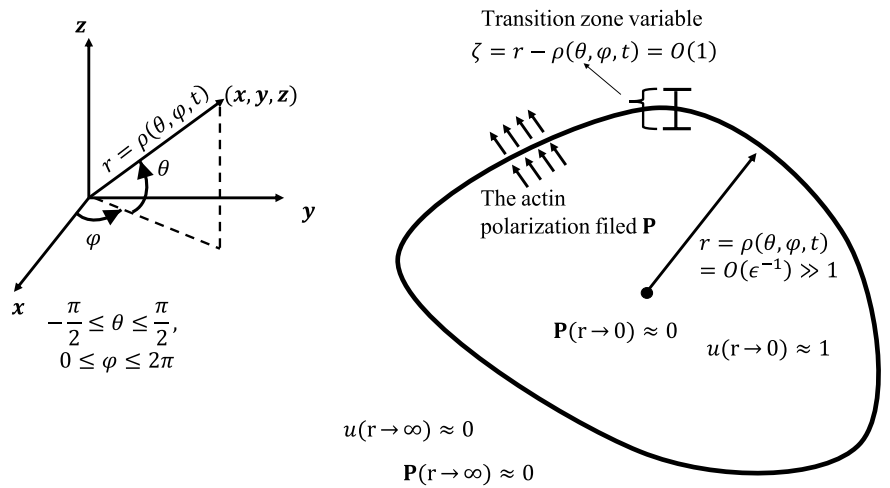

FiguRE 1. A schematic description of the 3D cell membrane dynamics in spherical coordinate system, and the boundary conditions of the simplified model $(2.1)-(2.5)$. The thickness of the cell wall (i.e., the width of the transition zone, where $u(r, t)$ is changed from nearly 1 to nearly 0 ) is $O(1)$, and the cell size is large. Therefore the ratio $\epsilon$ of the thickness of the cell wall to the size of the cell is small $\epsilon \ll 1$.

which has been suggested in [18]. Here $D_{u}$ is the stiffness of diffuse interface and $\alpha$ is the coefficient characterizing advection of $u$ by $\mathbf{P}$. The nonlinear term in (2.3) guarantees the existence of two stable homogeneous states, $u=0$ and $u=1$. The motion of the front between the phases $u=0$ and $u=1$ is governed by the value of $\delta$ : in the absence of $\mathbf{P}$, the front is motionless if $\delta=1 / 2$, the phase $u=1$ advances if $\delta<1 / 2$ and retreats if $\delta>1 / 2$. The suggested nonlocal expression for $\delta$ is

$$
\delta=\frac{1}{2}+\mu \tilde{V}-\sigma|\mathbf{P}|^{2}, \tilde{V}(t)=\int u \mathrm{~d}^{3} r-V_{0}
$$

where $\sigma$ is the contractility of actin filament bundle, $\mu$ is the stiffness of the volume constraint, and $V_{0}$ is the initial volume of the cell. According to (2.4), the generation of $\mathbf{P}$ diminishes $\delta$ and leads to the advancement of the cell membrane outwards. Also, the parameter $\delta$ provides a feedback control of the cell volume in a "soft way": the growth of the cell volume $\int u \mathrm{~d}^{3} r$ enhances $\delta$ braking that growth. According to the basic assumptions of the model,

$$
u(r \rightarrow \infty)=0, \quad 0<u<1,
$$

and $u$ is close to 1 inside the cell far from the cell membrane. It is assumed that the initial distribution of $u$ satisfies the conditions written above.

Note that all the parameters listed above are positive. Note also that the model (2.1)-(2.5) is nonlocal due to the definition of $\delta$.

We employ the spherical coordinate system,

$$
\begin{gathered}
(r, \theta, \varphi), 0<r<\infty,-\frac{\pi}{2}<\theta<\frac{\pi}{2}, 0<\varphi<2 \pi, \\
x=r \cos \theta \cos \varphi, y=r \cos \theta \sin \varphi, z=r \sin \theta
\end{gathered}
$$

(see Fig. 1). The interface $r=\rho(\theta, \varphi, t)$ is defined by the relation

$$
u(r=\rho(\theta, \varphi, t))=\frac{1}{2} .
$$


The three-dimensional polarization vector field, which describes the actin orientations, can be presented as $\mathbf{P}(r, \theta, \varphi, t)=p \hat{r}+q \hat{\theta}+w \hat{\varphi}$. Note that the directions of the unit vectors $\hat{r}, \hat{\theta}$ and $\hat{\varphi}$ depend on the angle coordinates $\theta$ and $\varphi$. In spherical coordinates,

$$
\nabla u=\hat{r} \partial_{r} u+\hat{\theta} \frac{\partial_{\theta} u}{r}+\hat{\varphi} \frac{\partial_{\varphi} u}{r \cos \theta}
$$

and

$$
\nabla^{2} u=\partial_{r}^{2} u+\frac{2 \partial_{r} u}{r}+\frac{1}{r^{2}}\left(\partial_{\theta}^{2} u-\tan \theta \partial_{\theta} u+\frac{\partial_{\varphi}^{2} u}{\cos ^{2} \theta}\right)
$$

The full expression for $\nabla^{2} \mathbf{P}$ is rather complex, but at large $r$,

$$
\nabla^{2} \mathbf{P}=\hat{r} \nabla^{2} p+\hat{\theta} \nabla^{2} q+\hat{\varphi} \nabla^{2} w+O\left(\frac{1}{r^{2}}\right)
$$

see, e.g., [5].

\section{Rotationally SYMmetriC CASE}

In the framework of the rotationally invariant model described above, the stationary cell is spherical. In the present section, we consider the perfectly spherical field, i.e., $u=u(r, t), \mathbf{P}=p(r, t) \hat{r}$, and $\rho=\rho(t)$.

Our basic assumption is that the radius of the cell $\rho(t)$ is large compared to the thickness of the transition zone, where $u(r, t)$ is changed from nearly 1 to nearly 0 . Therefore the ratio $\epsilon$ of the thickness of the cell wall to the size of the cell is small, $\epsilon \ll 1$, see Figure 1 .

In that case, the nonlocal term in (2.4) can be estimated as

$$
\int u \mathrm{~d}^{3} r \approx \frac{4 \pi}{3} \rho^{3}(t)
$$

The model (2.1)-(2.5) takes the form,

$$
\begin{aligned}
& u_{t}=D_{u}\left(u_{r r}+\frac{2}{r} u_{r}\right)-(1-u)(\delta-u) u-\alpha u_{r} \cdot p, \\
& \delta(r, t)=\frac{1}{2}+\frac{4 \pi \mu}{3}\left(\rho^{3}(t)-\rho_{0}^{3}\right)-\sigma p^{2}, \\
& p_{t}=D_{p}\left(p_{r r}+\frac{2}{r} p_{r}-\frac{2}{r^{2}} p\right)-\tau^{-1} p-\beta u_{r}, \\
& u(r \rightarrow \infty)=0, \quad 0<u<1, \\
& p(r \rightarrow \infty)=0,
\end{aligned}
$$

where $\rho_{0}$ is the initial radius of the sphere.

The assumption $\epsilon \ll 1$ allows to distinguish between the inner layer,

$$
\zeta=r-\rho(t)=O(1)
$$

where the fields of $u(r, t)$ and $p(r, t)$ are not constant and should be found by solving the problem, and the outer regions $\zeta \gg 1$ and $-\zeta \gg 1$, where the fields of the variables are trivial: at $\zeta \gg 1, u(r, t)=0, p(r, t)=0$; at $-\zeta \gg 1, u(r, t)=1, p(r, t)=0$ (up to exponentially small terms that are neglected). 
We rescale the radius of the cell,

$$
\rho(t)=\epsilon^{-1} R(t)
$$

Within the inner layer,

$$
\frac{1}{r}=\frac{\epsilon}{R(t)}-\frac{\epsilon^{2} \zeta}{R^{2}(t)}+\ldots
$$

Also, we assume that the parameter $\delta$ is close to $1 / 2, \delta-1 / 2=O(\epsilon)$, therefore one can expect that the front between the phases $u(r, t)=1$ and $u(r, t)=0$ moves slowly with the velocity $d \rho / d t=O(\epsilon)$, hence $d R / d t=O\left(\epsilon^{2}\right)$. Our goal is the analysis of that slow motion of the already formed front, i.e., we do not consider the stage of its formation during the time interval $O(1)$. Therefore, we rescale also the time variable as

$$
\tilde{t}=\epsilon^{2} t
$$

Let us redefine the variables inside the inner layer,

$$
R(t)=\tilde{R}(\tilde{t}), \quad u(r, t)=\tilde{u}(\zeta, \tilde{t}), \quad p(r, t)=\tilde{p}(\zeta, \tilde{t})
$$

and reformulate the governing problem for functions $\tilde{u}(\zeta, \tilde{t}), \tilde{p}(\zeta, \tilde{t})$. The chain rule yields

$$
\partial_{t}=-\epsilon \tilde{R}_{\tilde{t}} \partial_{\zeta}+\epsilon^{2} \partial_{\tilde{t}}, \quad \partial_{r}=\partial_{\zeta}
$$

Later on, we drop all the tildes.

The scaled equations (3.1a)-(3.1c) are,

$$
\begin{aligned}
& -\epsilon R_{t} u_{\zeta}=D_{u}\left(u_{\zeta \zeta}+\frac{2 \epsilon}{R(t)} u_{\zeta}\right)-(1-u)(\delta-u) u \\
& -\alpha u_{\zeta} p+O\left(\epsilon^{2}\right) \\
& \delta=\frac{1}{2}+\frac{4 \pi \mu}{3} \epsilon^{-3}\left(R^{3}(t)-R_{0}^{3}\right)-\sigma p^{2}=\frac{1}{2}+\epsilon \delta_{1}, \\
& -\epsilon R_{t} p_{\zeta}=D_{P}\left(p_{\zeta \zeta}+\frac{2 \epsilon}{R(t)} p_{\zeta}\right)-\tau^{-1} p \\
& -\beta u_{\zeta}+O\left(\epsilon^{2}\right) .
\end{aligned}
$$

Matching the inner solution to the outer one described above, we obtain the boundary conditions:

$$
u(\zeta \rightarrow-\infty)=1, u(\zeta \rightarrow \infty)=0, p(\zeta \rightarrow \pm \infty)=0 .
$$

Because the motion of the front is influenced by its curvature $1 / R$, the term $R_{t} u_{\zeta}$ in (3.6a) should balance the curvature term $2 u_{\zeta} / R(t)$ in the same equation. That justifies the choice of the radius and time scaling (3.3), (3.5), respectively.

In order to get $\delta-1 / 2=O(\epsilon)$, we have to assume the following scaling of parameters:

$$
\frac{4 \pi \mu}{3} \epsilon^{-3}=\epsilon M, \sigma=\epsilon S
$$




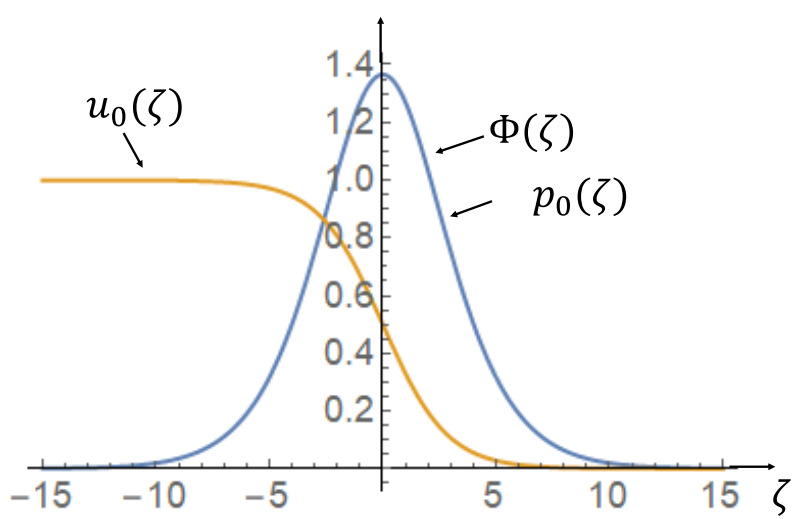

Figure 2. The plot of the kink solution (3.12a) of the order parameter $u_{0}(\zeta)$ and the functions $\Phi(\zeta)$ (also of $p_{0}(\zeta)$ when $\beta=1$ ) that describe the polarization field $(3.12 \mathrm{c})$. We employ the values of parameters $\tau=10, D_{u}=1, D_{p}=0.2, A=1, M=0.4, S=1.5$.

Also, we assume that

$$
\alpha=\epsilon A \text {. }
$$

Following to the scheme of the asymptotic approach, and introduce the expansions

$$
u=u_{0}+\epsilon u_{1}+\ldots, \quad p=p_{0}+\epsilon p_{1}+\ldots
$$

substitute (3.8), (3.9) and (3.10) into (3.6) and collect terms of the same order.

At the leading order, we obtain the following problem that describes the structure of the front:

$$
\begin{aligned}
& D_{u} u_{0 \zeta \zeta}=\left(1-u_{0}\right)\left(\frac{1}{2}-u_{0}\right) u_{0} \\
& D_{p} p_{0 \zeta \zeta}-\tau^{-1} p_{0}=\beta u_{0 \zeta} \\
& u_{0}(\zeta \rightarrow-\infty)=1, \quad u_{0}(\zeta \rightarrow \infty)=0, \\
& p_{0}(\zeta \rightarrow \pm \infty)=0 .
\end{aligned}
$$

Note that the obtained system does not contain derivatives with respect to the time variable: it describes the shape of the already formed transition zone. Problem (3.11) is identical to the corresponding problem obtained in the two-dimensional case in [4]: the structure of the one-dimensional domain wall does not depend on the dimension of the space. Therefore, we can use the solution of the problem obtained in [4]:

$$
\begin{aligned}
& u_{0}(\zeta)=\frac{1}{2}\left[1-\tanh \left(\frac{\zeta}{\sqrt{8 D_{u}}}\right)\right], \\
& p_{0}(\zeta)=\beta \Phi\left(\tau, D_{u}, D_{p}, \zeta\right), \\
& \Phi\left(\tau, D_{u}, D_{p}, \zeta\right) \\
& =\frac{1}{8} \sqrt{\frac{\tau}{2 D_{u} D_{p}}} \int_{-\infty}^{\infty} \mathrm{e}^{-|r| / \sqrt{\tau D_{p}}} \cosh ^{-2}\left(\frac{r-\zeta}{\sqrt{8 D_{u}}}\right) \mathrm{d} r .
\end{aligned}
$$

See Figure 2 for the plot of the fields in (3.12). 
At the order $O(\epsilon)$, we obtain a closed equation for $u_{1}$,

$$
\begin{gathered}
L\left[u_{1}\right]=\mathrm{RHS}, \\
L\left[u_{1}\right]=\left[D_{u} \partial_{\zeta \zeta}-\left(\frac{1}{2}-3 u_{0}+3 u_{0}^{2}\right)\right] u_{1} \text { and } \\
\mathrm{RHS}=\left(1-u_{0}\right) u_{0} \delta_{1}+A u_{0 \zeta} p_{0}-\frac{2 D_{u}}{R(t)} u_{0 \zeta}-R_{t} u_{0 \zeta}
\end{gathered}
$$

with boundary conditions

$$
u_{1}(\zeta \rightarrow \pm \infty)=0
$$

The second equation at the order $O(\epsilon)$ is

$$
D_{p} p_{1 \zeta \zeta}-\tau^{-1} p_{1}=\beta u_{1 \zeta}-R_{t} p_{0 \zeta}-\frac{2 D_{p}}{R} p_{0 \zeta}
$$

with boundary conditions

$$
p_{1}(\zeta \rightarrow \pm \infty)=0
$$

The problem (3.15), (3.16) is solvable for any right-hand side. As to the problem (3.13), (3.14), since $L\left[u_{0 \zeta}\right]=$ 0 , that problem is solvable only if its right-hand side is orthogonal to the solution of the homogeneous boundary value problem, $u_{0 \zeta}$, i.e.,

$$
\int_{-\infty}^{\infty} \mathrm{d} \zeta u_{0 \zeta} \cdot \mathrm{RHS}=0
$$

That solvability condition provides the closed evolution equation for the cell radius $R(t)$.

Using expressions

$$
\int_{-\infty}^{\infty} \mathrm{d} \zeta u_{0 \zeta}^{2}=\frac{1}{6 \sqrt{2 D_{u}}}, \quad \int_{-\infty}^{\infty} \mathrm{d} \zeta u_{0 \zeta} u_{0}\left(1-u_{0}\right)=-\frac{1}{6}
$$

we obtain the evolution equation for the radius of a spherical cell. Hence equation (3.17) may be written in the form,

$$
\begin{aligned}
& \frac{1}{\sqrt{2 D_{u}}}\left(R_{t}+\frac{2 D_{u}}{R}\right)=M\left[R_{0}^{3}-R^{3}(t)\right]+\Omega(\beta) \\
& \Omega(\beta)=6 \beta\left(A \Omega_{1}-\beta S \Omega_{2}\right),
\end{aligned}
$$

where,

$$
\begin{aligned}
& \Omega_{1}\left(\tau, D_{u}, D_{p}\right)=\int_{-\infty}^{\infty} \Phi(\zeta) u_{0 \zeta}^{2} \mathrm{~d} \zeta, \\
& \Omega_{2}\left(\tau, D_{u}, D_{p}\right) \\
& =\int_{-\infty}^{\infty} \Phi^{2}(\zeta)\left(1-u_{0}\right) u_{0} u_{0 \zeta} \mathrm{d} \zeta<0 .
\end{aligned}
$$




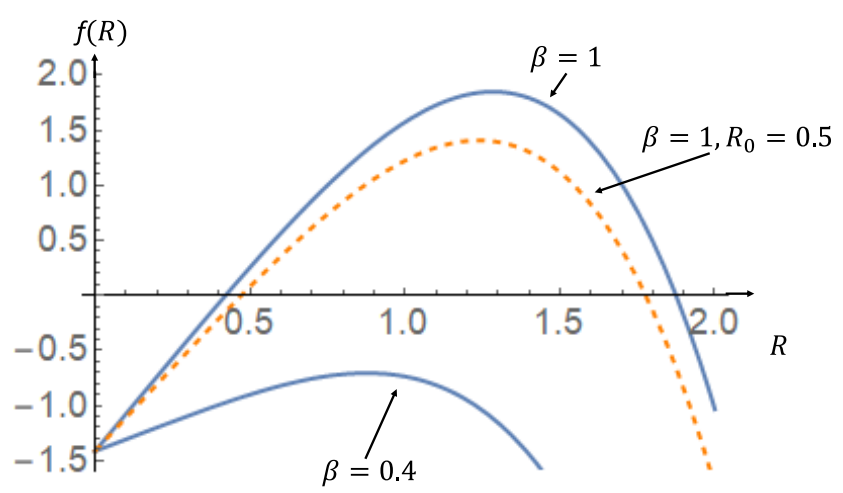

Figure 3. Solid lines present the plots of the function $f(R)$ in (3.22a), (3.22b) for the values $R_{0}=1$, and $\beta=1$ that manifest stable and unstable states, and for the value $\beta=0.4$ that is nonphysical since it does not include any steady states. We use the same values of parameters as in Figure 2. The dashed line is for the case $\beta=1$, and $R_{0}=0.5$.

Equation (3.19) is similar to that obtained in [4] for a two-dimensional circular cell. The differences are the coefficient 2 in the term proportional to $1 / R$, and the third power of the radius i.e., $R^{3}$, replacing $R^{2}$ in the term controlling the size of the cell. It is convenient to present equation (3.19) in the form

$$
\begin{aligned}
& a R_{t}=\frac{f(R)}{R}, \\
& f(R)=-M R^{4}+\left[M R_{0}^{3}+\Omega(\beta)\right] R-\sqrt{2 D_{u}},
\end{aligned}
$$

where $a=1 / \sqrt{2 D_{u}}$, which can be considered as a "force-velocity relation" for the surface of the motionless 3D cell immersed in 3D ECM (see [6]).

Note that $\Omega_{1}$ is positive and $\Omega_{2}$ is negative, therefore, $\Omega(\beta)$ is a monotonically growing function of $\beta$, see (3.20), (3.21). The nonlinearity of the dependence of $\Omega$ on $\beta$ is caused by the nonlinear term $-\sigma p^{2}$ in the expression (3.6b) for $\delta$. The physical origin of that term is the contraction of actin filament bundles.

In Figure 3 we plot the function $f(R)$ for two values of $\beta$; the graph shows the existence of two stationary radii, stable, $R_{+}(\beta)$ and unstable, $R_{-}(\beta)$, for $\beta=1$, while no stationary states for $\beta=0.4$. One can conclude that there exists a critical value $\beta_{c}$ such that there are no stationary solutions when $\beta<\beta_{c}$. In Appendix A we perform a detailed analysis for calculating $\beta_{c}$. We find that $\beta_{c}=0.637$ for the chosen set of parameters indicated in the caption of Figure 2, which is different from the value 0.41 found in the two-dimensional case [4].

The decrease of the cell radius (in the language of the phase-field model, the transition of the phase $u=1$ into the phase $u=0$ ) is caused by negative terms in the right-hand side of (2.3), (3.1a), among them the term $-\delta(1-u) u$, which is negative at large $R$, and by the diffusion term, which creates an effective "surface tension" of the cell surface. The positive term $-\alpha u_{r} p$ hinders the decrease of the cell radius. If $\beta$ is not sufficiently large, the polarization is not strong enough to stop the collapse of the cell. In that case, the cell shrinks until it disappears (see Fig. $3, \beta=0.4$ ). Of course, such values of $\beta$ have no biological meaning.

\section{Evolution of General SHAPE INTERFACE}

In this section, we apply the approach similar to that used in the previous section but for a general cell shape. We consider the field depending on the azimuthal and polar variables: $u=u(\zeta, \theta, \phi, t), p=p(\zeta, \theta, \phi, t)$, 
$R=R(\theta, \phi, t)$. We employ the scaling and definitions of the previous section. Following the chain rule, we calculate the derivatives using the following expressions for the differential operators:

$$
\begin{aligned}
\partial_{\theta}= & -\epsilon^{-1} R_{\theta} \partial_{\zeta}+\partial_{\theta}, \partial_{\varphi}=-\epsilon^{-1} R_{\varphi} \partial_{\zeta}+\partial_{\varphi} \\
\partial_{\theta}^{2}= & \epsilon^{-2} R_{\theta}^{2} \partial_{\zeta}^{2}-\epsilon^{-1}\left(R_{\theta \theta} \partial_{\zeta}+2 R_{\theta} \partial_{\zeta \theta}^{2}\right)+\partial_{\theta}^{2} \\
\partial_{\varphi}^{2}= & \epsilon^{-2} R_{\varphi}^{2} \partial_{\zeta}^{2}-\epsilon^{-1}\left(R_{\varphi \varphi} \partial_{\zeta}+2 R_{\varphi} \partial_{\zeta \varphi}^{2}\right)+\partial_{\varphi}^{2}, \\
\frac{1}{r} \partial_{\theta}= & -\frac{R_{\theta}}{R} \partial_{\zeta}+O(\epsilon), \quad \frac{1}{r^{2}} \partial_{\theta}=-\epsilon \frac{R_{\theta}}{R^{2}} \partial_{\zeta}+O\left(\epsilon^{2}\right), \\
\frac{1}{r} \partial_{\varphi}= & -\frac{R_{\varphi}}{R} \partial_{\zeta}+O(\epsilon), \quad \frac{1}{r^{2}} \partial_{\varphi}=-\epsilon \frac{R_{\varphi}}{R^{2}} \partial_{\zeta}+O\left(\epsilon^{2}\right), \\
\nabla^{2} u= & \left(1+\frac{R_{\theta}^{2}}{R^{2}}+\frac{R_{\varphi}^{2}}{R^{2} \cos ^{2} \theta}\right) u_{\zeta \zeta}+\epsilon\left[\left(\frac{2}{R}+\frac{R_{\theta}}{R^{2}} \tan \theta-\frac{R_{\theta \theta}}{R^{2}}-\frac{R_{\varphi \varphi}}{R^{2} \cos ^{2} \theta}\right) u_{\zeta}\right. \\
& \left.-\frac{2}{R^{2}}\left(R_{\theta} u_{\zeta \theta}+\frac{R_{\varphi}}{\cos ^{2} \theta} u_{\zeta \varphi}\right)-\frac{2 \zeta}{R^{3}}\left(R_{\theta}^{2}+\frac{R_{\varphi}^{2}}{\cos ^{2} \theta}\right) u_{\zeta \zeta}\right]+O\left(\epsilon^{2}\right) .
\end{aligned}
$$

The nonlocal term in (2.4) is approximated as follows,

$$
\int u \mathrm{~d}^{3} r \sim \frac{\epsilon^{-3}}{3} \int_{0}^{2 \pi} \mathrm{d} \varphi \int_{-\pi / 2}^{\pi / 2} R^{3}(\theta, \varphi, t) \cos \theta \mathrm{d} \theta .
$$

We use expansions (3.10), and define the auxiliary function $\Lambda$,

$$
\Lambda(\theta, \varphi, t)=\left(1+\frac{R_{\theta}^{2}}{R^{2}}+\frac{R_{\varphi}^{2}}{R^{2} \cos ^{2} \theta}\right)^{-1 / 2}
$$

At the leading order one finds,

$$
\begin{aligned}
& D_{u} \Lambda^{-2} u_{0 \zeta \zeta}=\left(1-u_{0}\right)\left(\frac{1}{2}-u_{0}\right) u_{0}, \\
& D_{p} \Lambda^{-2} p_{0 \zeta \zeta}-\tau^{-1} p_{0}=\beta u_{0 \zeta}, \\
& D_{p} \Lambda^{-2} q_{0 \zeta \zeta}-\tau^{-1} q_{0}=-\beta \frac{R_{\theta}}{R} u_{0 \zeta}, \\
& D_{p} \Lambda^{-2} w_{0 \zeta \zeta}-\tau^{-1} w_{0}=-\beta \frac{R_{\varphi}}{R \cos \theta} u_{0 \zeta},
\end{aligned}
$$

therefore similarly to the previous section one can calculate the solutions

$$
\begin{aligned}
& u_{0}(\zeta)=\frac{1}{2}\left[1-\tanh \left(\frac{\Lambda \zeta}{\sqrt{8 D_{u}}}\right)\right], \\
& p_{0}(\zeta)=\beta \Lambda \Phi(\Lambda \zeta) \\
& q_{0}(\zeta)=-\beta \frac{R_{\theta}}{R} \Lambda \Phi(\Lambda \zeta) \\
& w_{0}(\zeta)=-\beta \frac{R_{\varphi}}{R \cos \theta} \Lambda \Phi(\Lambda \zeta)
\end{aligned}
$$


The equation for $u$ at the order $O(\epsilon)$ have the form,

$$
\begin{aligned}
D_{u} \Lambda^{-2} u_{1 \zeta \zeta}-\left(\frac{1}{2}-3 u_{0}+3 u_{0}^{2}\right) u_{1} & =-R_{t} u_{0 \zeta}-D_{u}\left[\left(\frac{2}{R}+\frac{R_{\theta}}{R^{2}} \tan \theta-\frac{R_{\theta \theta}}{R^{2}}-\frac{R_{\varphi \varphi}}{R^{2} \cos ^{2} \theta}\right) u_{0 \zeta}\right. \\
& \left.-\frac{2}{R^{2}}\left(R_{\theta} u_{0 \zeta \theta}+\frac{R_{\varphi}}{\cos ^{2} \theta} u_{0 \zeta \varphi}\right)-\frac{2 \zeta}{R^{3}}\left(R_{\theta}^{2}+\frac{R_{\varphi}^{2}}{\cos ^{2} \theta}\right) u_{0 \zeta \zeta}\right] \\
& +A u_{0 \zeta}\left(p_{0}-\frac{R_{\theta}}{R} q_{0}-\frac{R_{\varphi}}{R \cos \theta} w_{0}\right)+\left(1-u_{0}\right) u_{0}\left\{\tilde{V}(t)-S\left(p_{0}^{2}+q_{0}^{2}+w_{0}^{2}\right)\right\},
\end{aligned}
$$

where the volume variation has the form,

$$
\tilde{V}(t)=M\left[\frac{1}{4 \pi} \int_{0}^{2 \pi} \mathrm{d} \varphi \int_{-\pi / 2}^{\pi / 2} R^{3}(\theta, \varphi, t) \cos \theta \mathrm{d} \theta-R_{0}^{3}\right] .
$$

We apply the solvability condition as in the previous section (3.17), which is the orthogonality of the equation's right-hand side to the solution of the homogenous equation $u_{0 \zeta}$, and obtain a closed form of the interface dynamics,

$$
a \Lambda R_{t}=-2 a D_{u} \mathcal{H}-\tilde{V}+\Omega
$$

where

$$
\mathcal{H}=\frac{1}{2} \nabla \cdot \hat{n}=\frac{1}{2} \nabla \cdot\left(\frac{\nabla(r-R)}{|\nabla(r-R)|}\right)
$$

is the mean local curvature of the surface $r=R(\theta, \varphi, t)$. For an explicit expression of the curvature see Appendix B. Note that in the spherically symmetric case $\Lambda=1, \mathcal{H}=1 / R$ and $\tilde{V}=M\left[R^{3}(t)-R_{0}^{3}\right]$. Therefore, equation (3.19) is recovered from equation (4.4).

Note that $\Omega$ in equation (4.4) depends on all the relevant parameters that describe the nonequilibrium molecular effects of the subcell level, see (3.20), and (3.21). Equation (4.4) is a closed evolutionary equation for the $3 \mathrm{D}$ cell interface dynamics at its onset of motion, which is an integro-differential equation, i.e., it is nonlocal, unlike that obtained in the spherical case (3.22a). For the details of the application of the solvability condition in order to obtain equation (4.4), we refer the reader to [4] and [3], where we perform similar calculations.

Notice that in (4.4) the expression $\Lambda R_{t}$ corresponds to the normal velocity $v_{n}$ of the interface, thus that equation is a generalization of the well-known curvature flow. By a proper scaling transformation, $t \rightarrow a^{2} t$ and $R(t) \rightarrow a R(t)$, the equation of motion of the cell boundary can be brought to a canonical form,

$$
v_{n}=-2 D_{u} \mathcal{H}-\tilde{V}+\Omega
$$

The obtained equation gives a relation between the quantities invariant with respect to any changes of the coordinate system: normal velocity, $v_{n}$, curvature, $\mathcal{H}$, integral expression $\tilde{V}$, and quantity $\Omega$ characterizing the natural velocity of a flat front in the absence of the volume constraint. It is not surprising that this relation is similar to the corresponding relation obtained in the two-dimensional case.

In addition it suggests an answer for the unrevealed force - velocity relation for the actin network that was highlighted in [6] in the context of shape dynamics of a $2 \mathrm{D}$ cell.

Note that in the framework of the model used in this paper, the surface tension of the cell and the characteristic width of the interface are determined by the same parameter, the interface stiffness parameter $D_{u}$. The effective surface tension can be changed by a modification of the model [2] . 


\subsection{Stability of the radial interface}

Let our base radial solution in (3.22a) be perturbed

$$
R=\bar{R}(t)+\hat{R}(\theta, \varphi, t), \quad \hat{R} \ll \bar{R} .
$$

The linearization of (4.4) around that base solution yields,

$$
\hat{R}_{t}=\frac{D_{u}}{\bar{R}^{2}(t)}\left(2 \hat{R}+\hat{R}_{\theta \theta}-\hat{R}_{\theta} \tan \theta+\frac{\hat{R}_{\varphi \varphi}}{\cos ^{2} \theta}\right)-\frac{3 M \bar{R}^{2}}{4 \pi a} \int_{0}^{2 \pi} \mathrm{d} \varphi \int_{-\pi / 2}^{\pi / 2} \hat{R} \cos \theta \mathrm{d} \theta .
$$

The solution has been expanded into a series over spherical harmonics,

$$
\hat{R}=\sum_{n=0}^{\infty} \sum_{m=-n}^{n} T_{m n}(t) P_{n}^{m}(-\sin \theta) e^{i m \varphi} .
$$

Recall that the associated Legendre polynomial $\Theta(\theta)=P_{n}^{m}(-\sin \theta)$ satisfies the equation

$$
\Theta^{\prime \prime}-\tan \theta \Theta^{\prime}+\left(n(n+1)-\frac{m^{2}}{\cos ^{2} \theta}\right) \Theta=0,
$$

and

$$
\int_{0}^{2 \pi} \mathrm{d} \varphi \int_{-\pi / 2}^{\pi / 2} \Theta(\theta) \cos \theta \mathrm{d} \theta=0
$$

unless $m=n=0$.

Substituting expansion (4.8) into (4.7) and taking into account the mutual orthogonality of spherical harmonics, we find:

$$
T_{m n}^{\prime}(t)=\left\{\frac{[2-n(n+1)] D_{u}}{\bar{R}^{2}(t)}-\delta_{m, 0} \delta_{n, 0} \frac{3 M \bar{R}^{2}(t)}{a}\right\} T_{m n}(t)=0 .
$$

For $n \neq 0$, the ratio

$$
\frac{T_{m n}^{\prime}(t)}{T_{m n}(t)}=\frac{[2-n(n+1)] D_{u}}{\bar{R}^{2}(t)}
$$

does not depend on $m$. For $n=1$, which corresponds to the spatial translation of the sphere as a whole, we find that $T_{m 1}(t)=$ const, $m= \pm 1,0$. Disturbances with $n \geq 2$, which describe the shape distortions, decay with time.

In the case $n=m=0$, which corresponds to a change of the sphere radius, we find that

$$
\frac{T_{00}^{\prime}(t)}{T_{00}(t)}=\frac{2 D_{u}}{\bar{R}^{2}(t)}-\frac{3 M \bar{R}^{2}(t)}{a}
$$

Using notation (3.22b), we can rewrite (4.9) as

$$
T_{00}^{\prime}(t)=\left.\sqrt{2 D_{u}} \frac{\mathrm{d}}{\mathrm{d} R}\left[\frac{f(R)}{R}\right]\right|_{R=\bar{R}(t)} T_{00} .
$$


The analysis of the sign of the expression in the right-hand side of (4.10) confirms the result obtained in Section 3: solution $R=R_{-}$is unstable and solution $R=R_{+}$is stable with respect to the radius change: when $\bar{R}(t)$ approaches its stationary value $R=R_{+}$, the derivative $d(f(R) / R) / d r$ at $R=\bar{R}(t)$ becomes negative (see Fig. 3), hence the spherical cell is stable with respect to spherical disturbances. Note that these results qualitatively are similar to the $2 \mathrm{D}$ case [4], where we find that the circular cell shape is stable concerning a small disturbance. Quantitatively, the evolution of the shape of a three-dimensional cell is slower.

\section{Conclusion}

We perform the analysis of a minimal phase field model that is a 3D generalization of the 2D model developed and investigated numerically in [16, 18], and [17] (a similar analysis of the latter model was done in [4]). In this model the order parameter $u$ is coupled with $3 \mathrm{D}$ polarization (orientation) vector field $\mathbf{P}$ of the actin network. In our opinion, that model can be applied for the description of the actin based protrusions of a 3D cell in 3D ECM [1].

We considered the rotational symmetric case, i.e., spherical shape interface, where we obtained a closed ordinary differential equation describing the evolution of the radius (3.22a). We found the minimum value $\beta_{c}$ for the actin creation that is compatible with the existence of a stationary cell solution (A.1). We found that when $\beta_{c}<\beta$, the circular cell can have some stationary radius, while in the case $\beta \leq \beta_{c}$ the cell shrinks until it disappears, which is meaningless in the context of cell dynamics. Also, we considered the general shape 3D cell dynamics. We found the leading order solutions, (4.2), and derived a closed integro-differential equation (4.4) governing the 3D cell dynamics, which includes the normal velocity of the membrane, curvature, volume relaxation rate, and a parameter $\Omega$ determined by the molecular effects of the subcell level. This result is similar to the $2 \mathrm{D}$ case [4].

We found an equation of motion of the cell interface at the onset of cell motion when the cell does not moves as a whole, which can be written in the canonical form (4.6).

The stability analysis shows that the non-spherical shape and the motion of the cell as a whole cannot appear due to the development of a linear instability of the spherical cell with the spherically symmetric polarization field localized near the cell boundary. In the framework of the considered model, the transition to a non-spherical shape needs a finite-amplitude disturbance significantly changing the polarization field inside the cell. That prediction has a clear biological meaning: the cell motion cannot be activated by an arbitrary weak fluctuation. The analysis of the motion's excitation, which can be carried out only numerically, is beyond the scope of the present paper.

\section{Appendix A.}

In this appendix we perform a detailed analysis for calculating $\beta_{c}$. The critical value $\beta_{c}$ has to satisfy three constraints: (i) $M R_{0}^{3}+\Omega\left(\beta_{c}\right)>0$, which guarantees the existence of maximum of $f(R)$ at a certain $R=R_{*}$, (ii) $f\left(R_{*}, \beta_{c}\right)=0$, (iii) $f_{R}^{\prime}\left(R_{*}, \beta_{c}\right)=0$ (see Fig. A.1(b)). As a result we find that $\beta_{c}$ is the positive solution of the quadratic equation

$$
M R_{0}^{3}+\Omega\left(\beta_{c}\right)=2^{\frac{19}{8}} 3^{\frac{-3}{4}} D^{\frac{3}{8}} M^{\frac{1}{4}}
$$

that can be found explicitly:

$$
\beta_{c}=\frac{1}{2 S \Omega_{2}}\left[A \Omega_{1}-\sqrt{\left(A \Omega_{1}\right)^{2}-S \Omega_{2}\left(2^{\frac{27}{8}} 3^{\frac{-7}{4}} D^{\frac{3}{8}} M^{\frac{1}{4}}-\frac{2}{3} M R_{0}^{3}\right)}\right]
$$

For $\beta=\beta_{c}$,

$$
R_{-}\left(\beta_{c}\right)=R_{+}\left(\beta_{c}\right)=R_{*}=2^{\frac{1}{8}} 3^{\frac{-1}{4}} D^{\frac{1}{8}} M^{\frac{-1}{4}}
$$


(a)

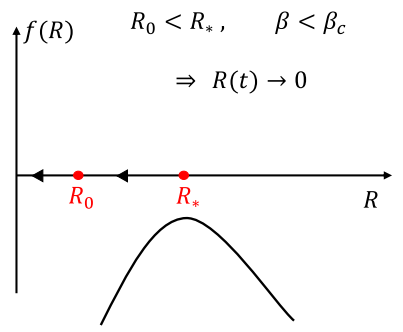

$(d)$ (b)

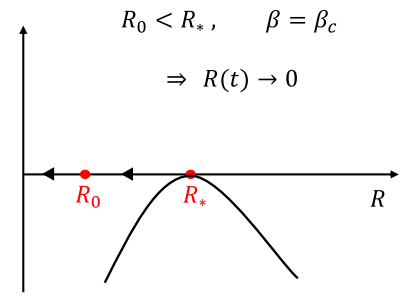

(c)

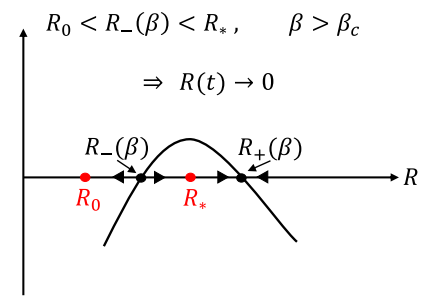

(e)

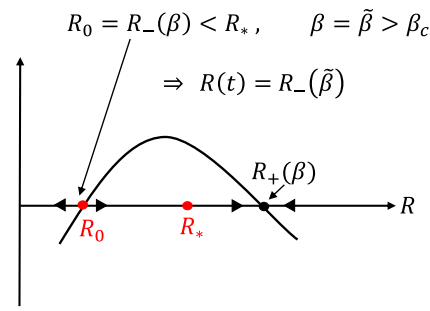

$R_{-}(\beta)<R_{0}<R_{*}, \quad \beta>\tilde{\beta}>\beta_{c}$

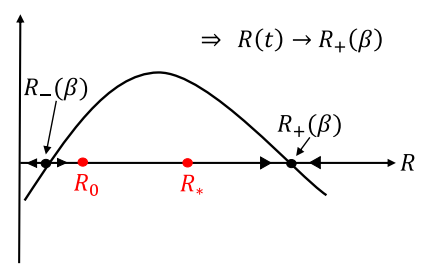

Figure A.1. A schematic description of the radius dynamics $R(t)$ that is governed by the ODE (3.22a), for all the subcases of the case $R_{0}<R_{*}$ as explained in the text. Notice that the positions of $R_{0}$, and $R_{*}$ are fixed (red points) for all the values of $\beta>0$.

notice that $R_{*}$ does not depend on $R_{0}$; for values of parameters indicated in Figure $2, R_{*}=1.041$. Because $\Omega(\beta)$ is a monotonically growing function of $\beta, R_{-}(\beta)$ decreases with the growth of $\beta$, and therefore $R_{-}(\beta)<R_{*}$ for any $\beta>\beta_{c}$. On the contrary, $R_{+}(\beta)$ increases with the growth of $\beta$. For values of parameters indicated in Figure 2 and $R_{0}=1$, we find $\beta_{c}=0.637$.

If $\beta<\beta_{c}$, then $f(R)<0$ for any $R$, therefore the cell radius decreases with time and tends to zero during a finite time (see Fig. A.1(a)). The temporal evolution of $R(t)$ in the case of $\beta>\beta_{c}$ depends on the relation between the initial radius $R_{0}$ and $R_{-}(\beta)$. If $R_{0}>R_{-}(\beta)$, then $R(t) \rightarrow R_{+}(\beta)$ as $t \rightarrow \infty$; if $R_{0}<R_{-}(\beta)$, then $R(t) \rightarrow 0$ during a finite time. Therefore, if $R_{0}>R_{*}, R(t)$ tends to a finite value for any $\beta>\beta_{c}$, because $R_{-}(\beta)<R_{*}<R_{0}$. However, if $R_{0}<R_{*}$, the cell shrinks even at $\beta>\beta_{c}$, if $\beta$ is still less than a certain value $\tilde{\beta}$ (see Fig A.1(c)), which is determined by the relation $R_{0}=R_{-}(\tilde{\beta})$ (see Fig. A.1 $(\mathrm{d})$ ). Because $f\left(R_{0}, \tilde{\beta}\right)=0$, the value of $\tilde{\beta}$ can be found by solving the equation,

$$
\Omega(\tilde{\beta})=\frac{\sqrt{2 D_{u}}}{R_{0}} .
$$

For $\beta>\tilde{\beta}, R_{-}(\beta)<R_{0}$, therefore the cell radius tends to a finite value (see Fig. A.1(e)).

In Figure A.2 we present the numerical solution of the ODE (3.22a) for the values $\beta=1$. As we can see, the cell radius can increase monotonically until it reaches the steady state value. This is because in the framework of our model, the volume is not conserved and influences the dynamics through the parameter $\delta(r, t)$, see equation (3.6b).

Note that at large $\beta$ (see Fig. A.1(e)) we have $\Omega(\beta) \gg 1$, and $R_{-}(\beta) \ll 1$, therefore one can approximate the expression of $f(R)$ in $(3.19)$ as:

$$
R_{-}(\beta) \approx \frac{\sqrt{2 D_{u}}}{\Omega(\beta)}
$$

We can see that for sufficiently large $\beta, R_{-}(\beta)$ can be arbitrary small. Therefore, for arbitrary small $R_{0}$, there exists such $\tilde{\beta}$ that the cell radius tends to a finite value, if $\beta>\tilde{\beta}$. 


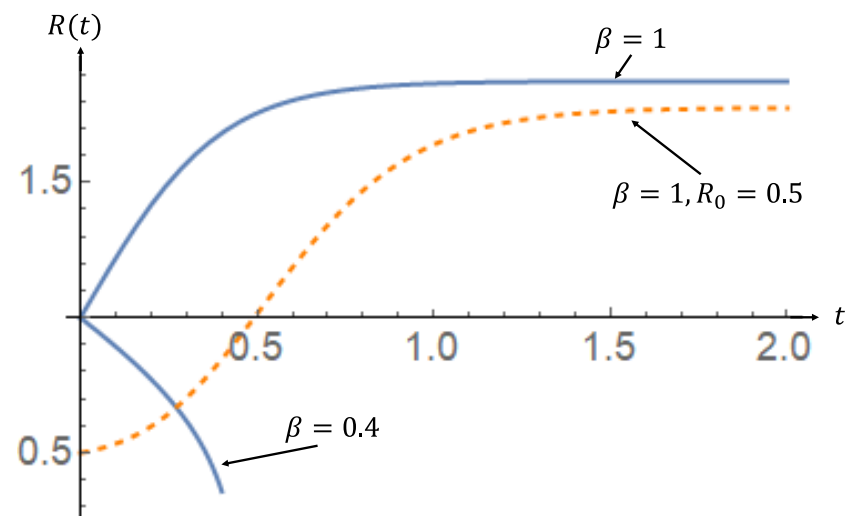

Figure A.2. Solid lines: numerical solutions of the ODE (3.22a) that yields $R(t)$ for values $R_{0}=1, \beta=1$ that manifest the existence of stationary radius, and for the value $\beta=0.4$ is below the critical value $\beta_{c}=0.637$ that is meaningless in the context of cell dynamics. We use the same values of parameters as in Figure 2. The dashed line is for the case $\beta=1$, and $R_{0}=0.5$.

For values of parameters indicated in Figure 2 and $R_{0}=0.5, \tilde{\beta}=0.96$. In Figures 3 and A.2, we show the numerical plots for the parameters $\beta=1$, and $R_{0}=0.5$.

Let us emphasize that the described effect is not physical: for such values of parameters, the model does not reflect the true behavior of cells.

\section{Appendix B.}

Here we give an explicit expression for the mean curvature of a surface given in spherical coordinate description $r=R(\theta, \varphi, t)$, see Figure 1. Following the definition (4.5), (4.1), one can calculate,

$$
\begin{aligned}
\nabla \cdot \hat{n}= & \Lambda\left\{\frac{2}{R}-\frac{R_{\theta \theta}}{R^{2}}+\frac{R_{\theta} \tan \theta}{R^{2}}-\frac{R_{\varphi \varphi}}{R^{2} \cos ^{2} \theta}\right\} \\
& +\Lambda^{3}\left\{\frac{1}{R^{3}}\left(R_{\theta}^{2}+\frac{R_{\varphi}^{2}}{\cos ^{2} \theta}\right)+\frac{R_{\theta}^{2} R_{\theta \theta}}{R^{4}}+\frac{2 R_{\theta} R_{\varphi} R_{\theta \varphi}}{R^{4} \cos ^{2} \theta}+\frac{R_{\varphi}^{2}}{R^{4} \cos ^{4} \theta}\left(\frac{1}{2} R_{\theta} \sin 2 \theta+R_{\varphi \varphi}\right)\right\}
\end{aligned}
$$

\section{REFERENCES}

[1] P.T. Caswell and T. Zech, Actin-based cell protrusion in a 3d matrix. Trends Cell Biol. 28 (2018) 823-834.

[2] R. Folch, J. Casademunt and A. Hernandez-Machado, Phase-field model for Hele-Shaw flows with arbitrary viscosity contrast. I. theoretical approach. Phys. Rev. E 60 (1999) 1724.

[3] M. Abu Hamed and A. Nepomnyashchy, Dynamics of curved fronts in systems with power-law memory. Physica D 328-329 (2016) $1-8$.

[4] M. Abu Hamed and A. Nepomnyashchy, A simple model of keratocyte membrane dynamics: the case of motionless living cell. Physica D 408 (2020) 132465.

[5] J. Happel and H. Brenner, Low Reynolds number hydrodynamics. Martinus Nijhoff Publisher (1983).

[6] K. Keren, Z. Pincus, G.M. Allen, E.L. Barnhart, G. Marriott, A. Mogilner and J.A. Theriot, Mechanism of shape determination in motile cells. Nature 453 (2008) 475-480.

[7] M.H. Mai and B.A. Camley, Hydrodynamic effects on the motility of crawling eukaryotic cells. Soft Matter 16 (2020) $1349-1358$.

[8] P.K. Mattila and P. Lappalainen, Filopodia: molecular architecture and cellular functions. Nature Publishing Group 9 (2008) 446-454.

[9] A. Mogilner, Mathematics of cell motility: have we got its number?. Math. Biol. 58 (2008) 105-134.

[10] A. Mogilner, E.L. Barnhart and K. Keren, Experiment, theory, and the keratocyte: an ode to a simple model for cell motility. Seminars in Cell Dev. Biol. 100 (2020) 143-151. 
[11] D.K. Schlüter, I.R. Conde and M.A.J. Chaplain, Computational modeling of single-cell migration: the leading role of extracellular matrix fibers. Biophys. J. 103 (2012) 1141-1151.

[12] E. Tjhung, A. Tiribocchi, D. Marenduzzo and M. Cates, A minimal physical model captures the shapes of crawling cells. Nat. Commun. 6 (2015) 5420.

[13] B. Winkler, I.S. Aranson and F. Ziebert, Confinement and substrate topography control cell migration in a $3 \mathrm{~d}$ computational model. Commun. Phys. 2 (2019) 82.

[14] P.H. Wu, D.M. Gilkes and D. Wirtz, Annual review of biophysics: the biophysics of 3d cell migration. Annu. Rev. Biophys. 47 (2018) 549-567.

[15] M.H. Zaman, R.D. Kamm, P. Matsudaira and D.A. Lauffenburger, Computational model for cell migration in three-dimensional matrices. Biophys. J. 89 (2005) 1389-1397.

[16] F. Ziebert and I.S. Aranson, Modular approach for modeling cell motility. Eur. Phys. J. Special Topics 223 (2014) $1265-1277$.

[17] F. Ziebert and I.S. Aranson, Computational approaches to substrate-based cell motility. npj Comput. Mater. 2 (2016) 16019.

[18] F. Ziebert, S. Swaminathan and I.S. Aranson, Model for self-polarization and motility of keratocyte fragments. J. R. Soc. Interface 9 (2012) 1084-1092.

\section{Subscribe to Open (S2O) A fair and sustainable open access model}

This journal is currently published in open access under a Subscribe-to-Open model (S2O). S2O is a transformative model that aims to move subscription journals to open access. Open access is the free, immediate, online availability of research articles combined with the rights to use these articles fully in the digital environment. We are thankful to our subscribers and sponsors for making it possible to publish this journal in open access, free of charge for authors.

\section{Please help to maintain this journal in open access!}

Check that your library subscribes to the journal, or make a personal donation to the $\mathrm{S} 2 \mathrm{O}$ programme, by contacting subscribers@edpsciences.org

More information, including a list of sponsors and a financial transparency report, available at: https://www.edpsciences.org/en/maths-s2o-programme 